\title{
Spectrally-Efficient Relay Selection with Limited Feedback
}

\author{
Ramy Tannious, Student Member, IEEE, and Aria Nosratinia, Senior Member, IEEE
}

\begin{abstract}
This paper addresses the multiplexing loss that occurs in relay networks due to causality of relays and the halfduplex constraint. We devise relay selection methods to recover the multiplexing loss in decode-and-forward (DF) relay networks, while requiring very little feedback (merely bits/relay). Two network topologies are studied: First the case is considered where a direct link is available between the source and destination, in addition to the relayed links. For this configuration, an incremental transmission scheme is proposed, and comprehensively analyzed, which uses limited feedback to improve both diversity as well as multiplexing gain. Then, the case without a direct link is considered, for which efficient non-orthogonal DF protocols are produced and analyzed. An interesting feature of the latter methods is unequal error protection capability via a family of embedded diversity-multiplexing (DMT) curves, which can be very useful for practical applications. Even considering this method's minimal DMT, a marked improvement over previous DF methods is observed, especially in high spectral efficiencies.
\end{abstract}

Index Terms-Cooperative diversity, diversity-multiplexing tradeoff, outage probability, relay selection.

\section{INTRODUCTION}

Relays can improve the performance of a wireless system via a number of mechanisms, such as increased spatial diversity or beamforming effects (whenever available). But for half-duplex relays, some time must be set aside for listening to the source, during which the relay must be silent. These silent times lead to a loss of spectral efficiency (also known as the multiplexing loss).

In this work, we address the issue of multiplexing loss in relay networks. As our main tool, we use variations on relay selection, which has nice properties but requires an exchange of channel state information between the nodes. We aim to recover the multiplexing loss using relay selection, under the constraint of very limited feedback (on the order of merely bits/relay).

Relay selection has been recently proposed to overcome some shortcomings of the existing relaying approaches in networks with multiple relays. Relay selection simplifies signaling, avoids complex synchronization schemes, and with careful design can preserve the spatial diversity provided by the total number of relays available in the network [1]. However, the selection process requires some information exchange between the relays and the destination which is considered an overhead. This overhead grows with the number of relays in the network.

The authors are with the Department of Electrical Engineering, The University of Texas at Dallas, Richardson, TX 75083-0688 USA, E-mail: ramy@student.utdallas.edu; aria@utdallas.edu. This work was supported in part by the NSF grant CNS-0435429. The material in this paper was presented in part at ICASSP 2008, Las Vegas, NV.

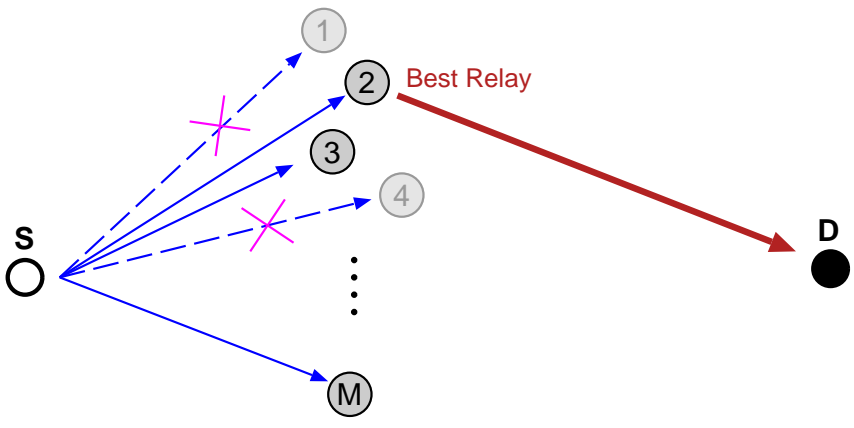

Fig. 1. Wireless network with relay selection. The best successful relay transmits to destination.

Moreover, in practice, the control channel that often conveys the feedback information is of very limited rate [2]. Hence, one is motivated to devise relay selection methods with limited feedback.

The setting of this work includes a source, a destination, and multiple relays, all of them single-antenna nodes in a fading wireless environment (see Figure 1). The relays employ a regenerative, decode-and-forward (DF) strategy. We consider two topologies: Either (a) the destination has a viable link to all transmitters, so a direct link from the source to destination exists, or (b) a direct link does not exist between the source and destination, so they can only communicate through the relays. The topologies include a very general inter-relay interference, which is not assumed to be either strong or weak. In fact, the general relay-on-relay interference is a key challenge and interesting facet of this work. Throughout this work, we define the best relay as the one with the highest instantaneous channel gain to the destination.

In the scenario where a direct source-destination link exists, one may use feedback not only to select the best relay, but also to select no relay when none is needed [3]. Thus, feedback can be used to gain both diversity as well as multiplexing. Motivated by this argument, we present an Incremental Transmission Relay Selection (ITRS) protocol, which employs a Type-I hybrid ARQ with packet combining at the destination, and includes a limited-feedback handshake for relay selection. We perform a detailed outage and DMT analysis of this method. ITRS meets the multiple-input-single-output (MISO) DMT bound, and provides a distinct improvement on a number of existing comparable methods.

In the scenario where a direct source-destination link does not exist, we employ overlapping (non-orthogonal) transmissions from the source to the relays and from the selected relay to the destination. This strategy leads to a Multi-Hop Relay 
Selection (MHRS) protocol. We analyze the performance of this method when the relays employ either successive cancellation, joint decoding of the incoming messages, or hybrid adaptive strategies. This system provides an embedded set of DMT curves that can be used for unequal-error protection (UEP), a very attractive feature for practical systems. Even the minimal DMT of this system is shown to improve on comparable existing methods.

We now outline some past works on DF multi-relay networks. To improve the spectral efficiency of relaying, Laneman and Wornell [4] propose distributed space-time codes (DSTC), which requires synchronization between the nodes. Azarian et al. [5] propose dynamic decode and forward (DDF) for multiple relays. The basic ideas of DDF are very nice, but unfortunately DDF does not scale with increasing number of relays in the high-rate regime. Bletsas et al. [1] propose an opportunistic relaying scheme that achieves the DMT of DSTC without the synchronization requirement, but requires transmit and receive-side channel-state information at the relays. Recently, Tajer and Nosratinia [6] show that it is possible to achieve the same DMT with very little information exchange.

Relay selection has also generated a sizable literature. The work in [7] shows the outage-optimality of relay selection under aggregate power constraint, which borrows much from the earlier work in [1]. In the multi-source, multi-destination scenarios, only a few works exist. Nosratinia and Hunter [8] demonstrate relay selection techniques that can capture maximum diversity in the number of cooperating nodes, while each node only knows its own receive channel state. Lin et. al. [9] presents relay selection criteria in the presence of node locations. Beres and Adve [10] considers various levels of centralization and compares selection with DSTC under instantaneous channel knowledge. There are also several works on relay selection adopt the amplify-and-forward (AF) scheme [11], [12], [13], [14], [15], [16] whose details are beyond the scope of this work.

There are some works with similarities to our ITRS protocol: Zhao and Valenti [3] were the first to consider hybridARQ in relays, but they select relays based on average channel gains, resulting in coding gain and second order diversity but not a diversity order that is equal to the number of available relays in the network. Lo et al. [17] propose a decentralized, limited-feedback, HARQ-based relay selection, and concentrate on BER and throughput studies.

Recently, Yang and Belfiore [18] present a sequential AF technique where, like our MHRS protocol, the relays transmit in succession. The results of [18] on AF networks cannot be directly compared with the present work, which is on DF networks. Furthermore, the achievable DMT of [18] is not known except for special cases where relays are isolated, or when two-slot transmission is used.

To summarize, the contributions of this paper are as follows: Relay selection methods are devised under very limited feedback and very general inter-relay interference conditions, for the purpose of recovering multiplexing gain in half-duplex DF relay networks. For two topologies with and without a direct link, we propose two protocols, named ITRS and MHRS, which are analyzed in detail and their DMT is provided or bounded. The MHRS protocol gives rise to an embedded set of DMT curves that can be used for unequal error protection. Our protocols improve over existing methods for half-duplex DF relays, including DSTC, DDF, and opportunistic relaying.

\section{System AND ChANNEL Models}

The system model consists of a destination node, a source, and $M$ half-duplex relays (see Figure 1). The channel gains between any two nodes is described by a flat, quasi-static Rayleigh fading model. We also consider the case where the source-destination link is non-existent, thus the communication must take place in a two-hop fashion through the relays, creating a bigger challenge for spectral efficiency. The analysis is general and avoids any special assumptions, such as isolated relays or strong inter-relay interference.

For relay selection, we assume the existence of a lowrate, reliable feedback from the destination to the relays (and possibly from destination to the source). Aside from this, no transmit-side channel state information (CSI) is assumed. The nodes have access to perfect receive CSI.

Throughout the paper, we assume that the input codewords are obtained from a random Gaussian codebook. The length of a codeword is asymptotically large but spans one coherence interval of the channel. Source and relay nodes each transmit under an average power constraint $P$. The receive noises are normally distributed $\sim \mathcal{N}\left(0, \sigma^{2}\right)$. The average receive SNR at each receiver is denoted $\rho$, i.e., $\rho=\frac{P}{\sigma^{2}}$. The system has $M$ relays, indexed $m=1, \ldots, M$. The channels between source and relays $\left(h_{s, m}\right)$, relays and destination $\left(h_{m, d}\right)$ and the inter-relay channels $\left(h_{m, m^{\prime}}\right)$ are zero-mean independent, circularly symmetric complex Gaussian random variables whose variances are $\lambda_{s, m}, \lambda_{m, d}, \lambda_{m, m^{\prime}}$, respectively. The magnitude square of channel coefficients, also known as effective channel gain, are denoted $g_{s, m}, g_{m, d}, g_{m, m^{\prime}}$ and follow exponential distributions. Whenever it exists, the source-destination channel is described with $h_{s, d}$ and follows similar statistics as the other links in the system. For simplicity of exposition, throughout the paper we assume that the source-relay channels have identical distributions, and the same holds for relaydestination and inter-relay channels, respectively. However, the DMT results do not depend on this assumption and continue to hold even if channels have non-identical (but finite) variance.

The performance of protocols is measured by outage [19], and the diversity-multiplexing tradeoff [20]. A channel is said to achieve multiplexing gain $r$ and diversity gain $d$ if there exists a sequence of codes $C(\rho)$ with rate $R(\rho)$ and resulting outage probability $P_{\text {out }}(\rho)$ such that:

$$
\lim _{\rho \rightarrow \infty} \frac{R(\rho)}{\log (\rho)}=r \quad \lim _{\rho \rightarrow \infty} \frac{\log P_{\text {out }}(\rho)}{\log (\rho)}=-d
$$

In the following developments, we say $f(\rho)$ is exponentially equal to $\rho^{v}$, denoted by $f(\rho) \doteq \rho^{v}$, if

$$
\lim _{\rho \rightarrow \infty} \frac{\log (f(\rho))}{\log (\rho)}=v
$$


1) The source transmits a packet.

2) If the destination correctly decodes the message, it broadcasts an ACK and system returns to Step 1. Otherwise destination broadcasts a NACK.

3) Upon receiving the NACK, the relays that successfully decoded the packet will declare their status via a one-bit packet (RTS - Request to Send) to the destination. The RTS packet includes a pilot.

4) The destination estimates channel gains, picks the best transmitter from among successful relays and the source, and broadcasts the index of the best node.

5) The best node will retransmit the packet. The destination combines its two received packets and decodes. If unsuccessful, destination is in outage.

Fig. 2. The Incremental Transmission with Relay Selection (ITRS) protocol

\section{INCREMENTAL TRANSMISSION RELAY SELECTION}

This section presents a protocol for a multi-relay network with limited feedback, called Incremental Transmission Relay Selection (ITRS). The network consists of a source, $M$ relays, and a destination, where the destination has a fading link to the source as well as the relays (see Section II). In this protocol, the limited feedback has dual use: it selects the best relay, thus improving diversity, and also enables retransmission (HARQ), thus improving spectral efficiency. The broad outline of the protocol is as follows: A packet is broadcast by the source. If the destination cannot decode, a limited-feedback handshake is performed that identifies the best available node (among source and relays), which will retransmit the packet. The ITRS protocol is described in detail in Figure 2. Note that the channel gains are assumed to remain fixed during steps 35 .

The ITRS protocol uses a maximum of one retransmission. Further retransmissions would reduce (and eventually eliminate) outage, but also incur further delay. We study the case of one retransmission, which incurs modest delay and yet captures the biggest part of the gains available through retransmissions.

The ITRS protocol uses type-I H-ARQ with packet combining, i.e., relays use the same codebook as the source. Type-II H-ARQ, where the relays use non-identical codebooks, has better mutual information but also increases complexity. The two methods achieve the same DMT.

The ITRS protocol includes the source in the competition for the re-transmission, thus improving the diversity as well as throughput, as seen in the sequel.

The protocols presented in this paper require feedback, whose transmission in turn requires a channel and a protocol. Feedback often goes through a control channel that exists in many wireless standards. The medium access layer for these channels can be either contention-based or slotted. In the former, all relays contend in sending their RTS to the destination, in which case the relay address (ID) must be attached to the RTS packet. In a time-slotted system, on the other hand, each relay transmits an RTS in its designated minislot only. This avoids collision between relays, but some minislots may go unused depending on the number of available relays, therefore usage of channel resources may be inefficient.

\section{A. Outage Probability and Effective Rate}

During the first transmission of a packet by the source, the received signals at the relays and the destination are given by:

$$
\begin{aligned}
\mathbf{y}_{m} & =h_{s, m} \mathbf{x}_{s}+\mathbf{z}_{m} \quad m=1, \ldots, M \\
\mathbf{y}_{d} & =h_{s, d} \mathbf{x}_{s}+\mathbf{z}_{d}
\end{aligned}
$$

During a re-transmission, the received signal at the destination is given by

$$
\mathbf{y}_{d}=h_{m^{*}, d} \mathbf{x}_{m^{*}}+\mathbf{z}_{d}
$$

Where $m^{*}$ denotes the index of the selected relay. We emphasize again that for the retransmission, the best relay is chosen from among all the nodes (including the source) that have possession of the packet data at that time.

During the original packet transmission, the mutual information across the source-destination channel is:

$$
I_{D}=\log \left(1+\rho g_{s, d}\right)
$$

If a retransmission occurs, the combination of the two transmissions forms an equivalent channel between the source and the destination, whose mutual information is:

$$
I_{\text {itrs }}^{*}=\frac{1}{2} \log \left[1+\rho\left(g_{s, d}+g_{m^{*}, d}\right)\right]
$$

Denote the set of all relays that have decoded the source message with $D(s)$. Using the law of total probability, the outage probability can be expressed as:

$$
\begin{aligned}
P_{\text {out }}= & \sum_{t=1}^{M+1} \operatorname{Pr}\left\{I_{\text {itrs }}^{*}<\frac{R}{2}\left|I_{D}<R,\right| D(s) \mid=t\right\} \\
& \times \operatorname{Pr}\left\{I_{D}<R\right\} \operatorname{Pr}\{|D(s)|=t\}, \\
= & \sum_{t}^{M+1} \operatorname{Pr}\left\{I_{\text {itrs }}^{*}<\frac{R}{2}|| D(s) \mid=t\right\} \operatorname{Pr}\{|D(s)|=t\}
\end{aligned}
$$

The outage probability in (8) is computed for a rate $R$ in case of successful source transmission and for a rate $\frac{R}{2}$ in case of incremental transmission due to information repetition.

The probability that exactly $t$ nodes (including the source) know the message is given by [4],

$$
\begin{aligned}
\operatorname{Pr}\{|D(s)|=t\}= & \left(\begin{array}{c}
M \\
t-1
\end{array}\right) \exp \left(-\frac{2^{R}-1}{\lambda_{s, m} \rho}\right)^{t-1} \\
& {\left[1-\exp \left(-\frac{2^{R}-1}{\lambda_{s, m} \rho}\right)\right]^{M-t+1} }
\end{aligned}
$$

By substituting (9) in (8) and obtaining the CDF of $I_{i t r s}^{*}$ one can find a closed form expression for the overall outage probability $(M \geq 1)$ :

$$
\begin{aligned}
P_{\text {out }, \text { ITRS }}= & \sum_{t=1}^{t=M+1} F_{W}(\gamma) \times\left(\begin{array}{c}
M \\
t-1
\end{array}\right) \exp \left(-\frac{\gamma}{\lambda_{s, m}}\right)^{t-1} \\
& \left(1-\exp \left(-\frac{\gamma}{\lambda_{s, m}}\right)\right)^{M-t+1}
\end{aligned}
$$


Where

$$
\begin{array}{r}
F_{W}(\gamma)=\left[t \sum _ { k = 1 } ^ { t - 1 } ( \begin{array} { c } 
{ t - 1 } \\
{ k }
\end{array} ) \frac { ( - 1 ) ^ { k } } { k } \left(1+\frac{\exp (-\mu(k+1) \gamma)-1}{(k+1)}\right.\right. \\
-\exp (-\mu \gamma))]+t(1-(\mu \gamma+1) \exp (-\mu \gamma))
\end{array}
$$

$\gamma=\frac{2^{R}-1}{\rho}$ and for simplicity we let $\lambda_{s, d}=\lambda_{m^{*}, d}=\frac{1}{\mu}$. The details of the analysis are carried out in Appendix I.

We now calculate the throughput $\eta$, also known as effective rate or expected rate, for the ITRS protocol. This value has two contributing terms: for packets that are received in one try, or two tries, as shown below:

$$
\begin{aligned}
\eta=R & \exp \left(-\frac{2^{R}-1}{\rho \lambda_{s, d}}\right)+\frac{R}{2}\left[\left(1-\exp \left(-\frac{2^{R}-1}{\rho \lambda_{s, d}}\right)\right)\right. \\
& \left.\left(1-P_{\text {out }}\right)\right]
\end{aligned}
$$

The first term is the average rate from the direct link and it occurs with the associated success probability. The second term is the average rate from HARQ with relay selection. Therefore, the rate is reduced to half since two blocks are used to transmit the same information. This second round of transmission is successful under the following two conditions:

1) The first round transmission failed.

2) The second round transmission with relay selection is successful.

We note that a somewhat similar notion of expected spectral efficiency was developed in [21] for a single-relay Amplify and Forward (AF) incremental relaying. The mapping $R \rightarrow \eta$ is highly nonlinear and one may choose $R$ to maximize the throughput $\eta$.

The ITRS protocol requires $1+\frac{\log (M+1)}{M+1}\left[1-\exp \left(-\frac{2^{R}-1}{\rho \lambda_{s, d}}\right)\right]$ bits of overhead per transmitting node. First, the destination broadcasts one bit of ACK/NACK. With probability $1-$ $\exp \left(-\frac{2^{R}-1}{\rho \lambda_{s, d}}\right)$, the response is a NACK. The available relays and the source will respond with one-bit (known as Request To Send, or RTS). Finally, the destination will broadcast the index of the best node via $\log (M+1)$ bits. Asymptotically, this overhead is one bit per node per packet.

The above overhead analysis only counts the information bits in the feedback/control channels. It does not include the extra overhead that must be included in practice, for example a preamble. We also note that although we strive to design protocols with minimal overhead, this overhead will not affect the DMT results. In the high SNR regime, any constant overhead will diminish with respect to the channel capacity.

Remark 1: If the source is excluded from the competition for relaying the expected rate will be given by

$$
\begin{gathered}
\eta=R \exp \left(-\frac{2^{R}-1}{\rho \lambda_{s, d}}\right)+\frac{R}{2}\left\{\left[1-\exp \left(-\frac{2^{R}-1}{\rho \lambda_{s, d}}\right)\right]\right. \\
\left.\left[1-\left(1-\exp \left(-\frac{2^{R}-1}{\rho \lambda_{m *, d}}\right)\right)^{M}\right]\left(1-P_{\text {out }}\right)\right\}
\end{gathered}
$$

This expected rate will approach (12) for large number of relays and high $\rho$.

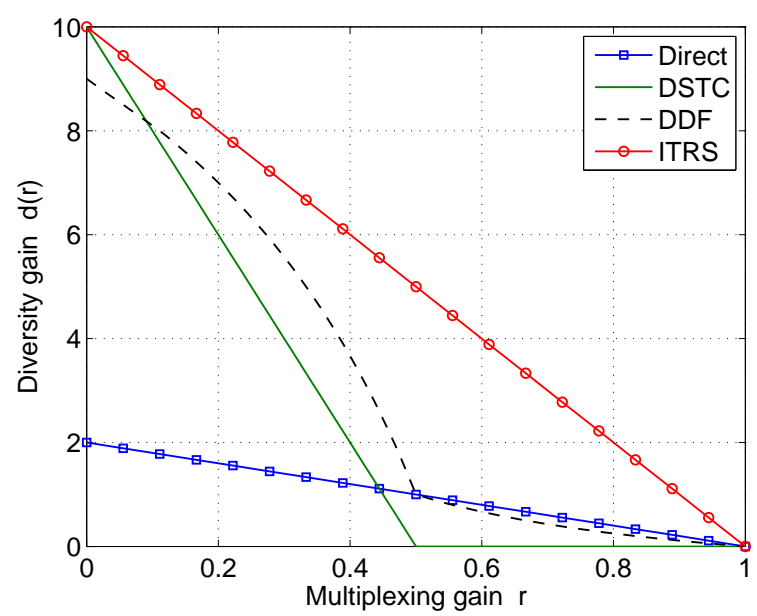

Fig. 3. Diversity-multiplexing tradeoff of ITRS compared with distributed space-time coding, dynamic decode-and-forward and HARQ non-cooperative transmission. There are eight relays and the source-destination link exists.

\section{B. DMT Analysis}

In the high-SNR regime the performance of ITRS is described as follows, where we denote $(\cdot)^{+}=\max \{\cdot, 0\}$.

Theorem 1: The ITRS protocol achieves the following diversity-multiplexing tradeoff:

$$
d_{\text {ITRS }}(r)=(M+2)(1-r)^{+}
$$

which is equivalent to the optimal DMT of a system with one source node and $M$ relay nodes [5], [20].

Proof: See Appendix II

Corollary 1: ITRS with independent codebooks (type-II ARQ) achieves the same DMT.

Proof: Since the identical codebooks achieve the MISO DMT bound, and independent codebooks will do no worse, then type-II ARQ will also achieve the same upper bound.

The DMT of ITRS protocol with eight relays is shown in Figure 3. Also shown are other DF-based protocols, including the DDF of Azarian et al. [5, Theorem 6], and the DSTC of Laneman-Wornell [4], which has DMT equivalent to Bletsas et al. [1]. For fairness, we have compared our algorithm with a slight enhancement of DSTC by allowing its source to participate in the second phase of transmission. For the non-cooperative benchmark, the DMT of HARQ signaling is shown, where a maximum diversity order of two is possible via packet combining [22, Corollary 3]. We see that ITRS has improved performance over previous protocols across all $r$, while requiring only limited feedback.

Protocol analysis corroborates the merits of allowing the source to compete for transmission in the relaying phase, which results in higher effective rate and diversity order $M+2$ (since $M+1$ nodes act as a distributed antenna array in the second phase).

Remark 2: Consider the case where the destination node is limited to a type-I HARQ without diversity combining. Then the ITRS protocol still works, and achieves a slightly diminished maximum diversity order of $M+1$. Thus, ITRS can also be used in networks with very simple nodes without packet combining capabilities, e.g., wireless sensor networks. 
1) The source transmits alone in the first time slot. Then, in each time slot:

2) Relays that successfully decode the source packet, declare their status to the destination via a one-bit RTS packet (which includes a pilot).

3) The destination picks the best relay and broadcasts its index.

4) The best relay retransmits its decoded packet, which the destination attempts to decode. At the same time, the source transmits a new packet.

5) The source packet and relayed packet combine at other relays. Relays attempt to decode new source packet in the presence of interference. Then continue to Step 2.

Fig. 4. The Multi-Hop with Relay Selection (MHRS) protocol.

Remark 3: When SNR is low, retransmissions are frequent. If, furthermore, relays are not abundant, the source may be called upon to re-transmit frequently, which is a strain on its power resources. Under these conditions, one may use a variation of ITRS, where the source will re-transmit only if all relays have failed to decode. This results in a slightly diminished maximal diversity of $M+1$, while extending the lifetime of the network.

\section{Two-Hop Relay SELECTION}

When a direct path between the source and destination is unavailable, the relays must repeat the signal in a two-hop fashion. But it has been well-known that repeating the source's transmission limits the spectral efficiency in relay networks. The work in this section shows that in the presence of multiple relays, one may recover a good part of the rate loss with appropriate protocol design. We present a Multi-Hop Relay Selection (MHRS) protocol with attractive spectral efficiency, using non-orthogonal decode-and-forward signaling. The basic operation of the algorithm is as follows: in each time interval, the source transmits a new packet for the benefit of the relays. Simultaneously, the "best" relay re-transmits a packet for the benefit of the destination, interfering with the reception of other relays. All relays attempt to decode in the presence of interference, to be able to participate in the next round of transmission. The details of the MHRS protocol is described in Figure 4. It is assumed the channel remains constant within steps $2-4$.

A sample timing diagram of the MHRS protocol is shown in Figure 5. The reception status of the relays is shown with a check or a cross. A check mark means successful decoding while a cross means failed decoding. Notice that whenever a relay transmits, due to the half-duplex constraint, it cannot receive. Therefore, in the following time interval, it is operating at a disadvantage since it cannot peel-off the interference signal from the source transmission. Thus, in this sample path many relays are shown to fail in decoding immediately after transmission. In each time interval, the best available relay is called upon to transmit to the destination. Note that at the very end, the source is silent while a relay communicates the final packet with the destination. The overhead for control in the MHRS protocol is $1+\frac{\log (M-1)-1}{M}$ bits per node per packet

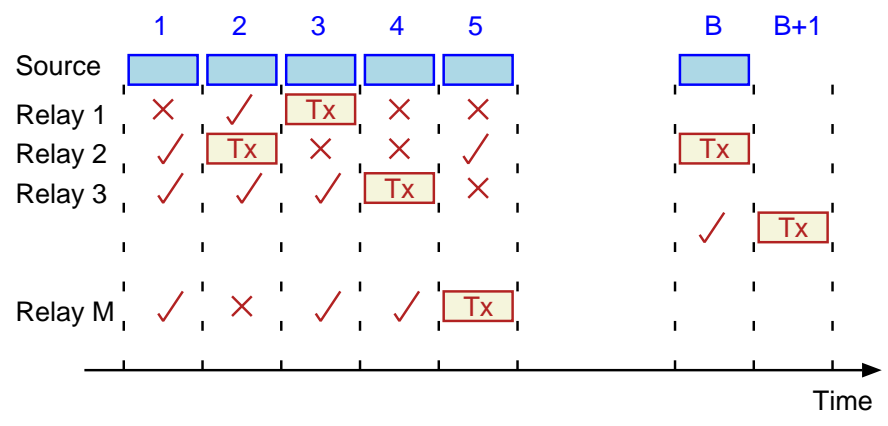

Fig. 5. Frame structure of MHRS protocol.

(as mentioned in the previous section, this overhead does not include preamble and pilots of packets transmitted through the control channel).

Remark 4: If no relay has successfully decoded the sources message, i.e. an empty decoding set, the source will notice the absence of the relays' RTS (Request to Send) signal, and can resend the packet. This is easy to implement, but in general not straight forward to analyze. The full analysis of this extension can be a subject of future work.

In the following, we calculate a DMT upper bound and then present two decoding protocols at the relays, each with their own achievable DMT. The first decoding protocol is based on successive cancellation at the relays. The key result in successive cancellation is that, after each relay's transmission, due to interference it cannot recover its own decoding diversity, thus it cannot contribute to the overall diversity any longer. It follows that across time, a family of DMT curves are produced with varying diversity. Every $B+1$ blocks, the maximal diversity is restored when the source transmits alone and relays are silent.

The interesting outcome of the family of DMT curves is that it allows variable error-protection. The overall data can be divided into several groups with varying error sensitivity. The most sensitive data is transmitted early, and enjoy the best DMT, while other packets with lower sensitivity are transmitted later. To our knowledge, this is the first formal introduction of a variable-error protection scheme in relay networks, in the DMT sense.

If the relays have enough computational power, they may be able to jointly decode the two interfering signals. We show that a hybrid strategy, incorporating both successive cancellation and joint decoding, in part meets the DMT upper bound, and is superior to successive cancellation.

\section{A. DMT Upper bound}

We upper bound the DMT of our system by considering a hypothetical system where the individual relays are replaced with one MIMO relay. This will result in a system that operates as follows: during each interval, the best antenna for the relay-destination channel is used for relaying, while the other antennas listen to the source to receive the next frame. Since the new system is equivalent to perfect information exchange between relays, its performance upper bounds the performance of our system. 
Using the above model, we have the following result:

Theorem 2: The DMT of the multi-hop with relay selection (MHRS) protocol is upper bounded by:

$$
d^{*}(r)=(M-1)\left(1-\frac{B+1}{B} r\right)^{+}
$$

Proof: According to [23, Lemma 1], the DMT of a channel with a single MIMO relay is bounded by the minimum of the source-relay and relay-destination DMT bounds. The source-relay DMT bound is the well-known SIMO bound

$$
d_{S R}^{*}(r)=(M-1)(1-r)^{+}
$$

The relay-destination DMT is bounded by a MISO DMT with single-antenna selection out of $M-1$ available antennas, which has been recently reported in [24, Theorem 4.1].

$$
d_{R D}^{*}(r)=(M-1)(1-r)^{+}
$$

The proof is completed by taking the minimum of the previous two bounds and taking into account the rate loss due to the causality of the relay. The optimal DMT is given by,

$$
d^{*}(r)=(M-1)\left(1-\frac{B+1}{B} r\right)^{+}
$$

\section{B. Successive Cancellation DMT and Variable-Error Protec- tion}

Based on the protocol description, the received signals at the intermediate nodes and the destination are respectively given by:

$$
\begin{aligned}
\mathbf{y}_{m} & =h_{s, m} \mathbf{x}_{s}+h_{m^{*}, m} \mathbf{x}_{m^{*}}+\mathbf{z}_{m} \\
\mathbf{y}_{d} & =h_{m^{*}, d} \mathbf{x}_{m^{*}}+\mathbf{z}_{d}
\end{aligned}
$$

Where $m=1, \ldots, M$ and $m \neq m^{*}$. The transmission of the packets occurs in cycles. The source packets in each cycle are indexed by $b=1, \ldots, B$. At the end of the cycle, the source stays silent for one period so that the last packet can be cleared to the destination. Then the entire process starts again (see Figure 5).

The mutual information of the channel between the "best" relay node and the destination is given by:

$$
I_{m h r s}^{*}=\log \left(1+\rho \max _{m \in D(s)} g_{m}\right)
$$

The outage probability can be expressed as:

$$
\begin{aligned}
\operatorname{Pr}\left\{I_{m h r s}^{*}<R\right\}= & \sum_{t} \operatorname{Pr}\{|D(s)|=t\} \\
& \operatorname{Pr}\left\{I_{m h r s}^{*}<R|| D(s) \mid=t\right\}
\end{aligned}
$$

Thus, the diversity-multiplexing tradeoff is governed by two probabilities: the relay decoding probability, and the outage probability conditioned on a decoding set $D(s)$.

Recall that from a relay's viewpoint, there are two packets arriving simultaneously over the air. For example, when the source is transmitting packet $b$, another relay is transmitting packet $b-1$. We aim to calculate the probability that a given relay fails to decode the source packet $b$, denoted $P\left(O_{b}\right)$.
Conditioned on having decoded packet $b-1$, the probability of relay outage for packet $b$ is:

$$
P\left(O_{b} \mid \bar{O}_{b-1}\right) \doteq \rho^{-(1-r)^{+}}
$$

However, if the previous packet $b-1$ cannot be peeled off, the interference has the same order of magnitude as the signal, and thus

$$
P\left(O_{b} \mid O_{b-1}\right) \doteq \rho^{0}
$$

Now, we derive a equation using the law of total probability

$$
\begin{aligned}
P\left(O_{b}\right) & =P\left(O_{b} \mid \bar{O}_{b-1}\right) P\left(\bar{O}_{b-1}\right)+P\left(O_{b} \mid O_{b-1}\right) P\left(O_{b-1}\right) \\
& \doteq \rho^{-(1-r)^{+}}\left[1-P\left(O_{b-1}\right)\right]+\rho^{0} P\left(O_{b-1}\right) \\
& =\rho^{-(1-r)^{+}}+P\left(O_{b-1}\right)\left[1-\rho^{-(1-r)^{+}}\right] \\
& \doteq \rho^{-(1-r)^{+}}+P\left(O_{b-1}\right) \quad \text { for } r<1
\end{aligned}
$$

During transmission of the first packet, the relays listen to the source signal without interference, so $P\left(O_{1}\right) \doteq \rho^{-(1-r)^{+}}$. Then according to the above recursion, each relay will continue to decode with $P\left(O_{b}\right) \doteq \rho^{-(1-r)^{+}}$until it is called upon to transmit. During a relay's transmission interval, it cannot listen to the source signal, so in the next interval, it will have to decode the source signal without knowledge of the interference. This task has outage probability proportional to $\rho^{0}$. From this point onwards, the recursion shows that the relay will continue to experience outage proportional to $\rho^{0}$. In other words, the loss of diversity propagates in time. The diversity of all relays is restored at the end of the transmission cycle.

Now consider $\operatorname{Pr}\{|D(s)|=t\}$. To have exactly $t$ decoding relays, $M-t$ relays must be in outage. For the first packet, all relays decode without interference on i.i.d. channels, therefore

$$
\operatorname{Pr}\{|D(s)|=t\} \doteq \rho^{-(M-t)(1-r)^{+}} \quad \text { for } b=1
$$

Subsequently, one of the relays is chosen to relay packet $b=1$. This relay will lose its diversity for all subsequent packets, until the end of the cycle. Each relay that transmits will then stay out of the decoding set in successive blocks with probability proportional to $\rho^{0}$. Effectively, as we go through the packets, the number of available relays is reduced one-byone. ${ }^{1}$ Thus, for packet $b$, the probability that there are $t$ relays ready to transmit is:

$$
\operatorname{Pr}\{|D(s)|=t\} \doteq \rho^{-(M-b+1-t)^{+}(1-r)^{+}}
$$

Now we look at the destination outage conditioned on the decoding set.

$$
\begin{aligned}
\operatorname{Pr}\left\{I_{m h r s}^{*}<R|| D(s) \mid=t\right\} & =\left(1-\exp \left(-\frac{2^{R}-1}{\rho \lambda_{m^{*}, d}}\right)\right)^{t} \\
& =\left(1-\exp \left(-\frac{\rho^{r-1}}{\lambda_{m^{*}, d}}\right)\right)^{t} \\
& \doteq \rho^{-t(1-r)^{+}}
\end{aligned}
$$

Substituting (24) and (25) in (22), we get:

$$
\operatorname{Pr}\left\{I_{m h r s}^{*}(b)<R\right\} \doteq \rho^{-(M-b+1)^{+}(1-r)^{+}}
$$

${ }^{1}$ Please note that it is possible for the relays to return to the decoding pool, as is shown in Figure 5, but not with probability-1 asymptotically with SNR. 
Finally, we have to take into account a fractional rate loss, because as seen in Figure 5, overall $B$ blocks are transmitted in $B+1$ time intervals, due to causality requirement of the relays. Therefore, we must make the adjustment $r \rightarrow \frac{B+1}{B} r$. The final result can be described as follows:

Theorem 3: For the MHRS protocol, the following diversity-multiplexing tradeoff is achievable for the packet $b$, where $b \in\{1, \ldots, B\}$.

$$
d(r, b)=(M-b+1)^{+}\left(1-\frac{B+1}{B} r\right)^{+}
$$

The variable-error protection strategy is an attractive feature of this system that allows a tailoring of transmission to the application requirements. Figure 6 shows the family of DMT's obtained in a MHRS protocol with five relays and $B=5$.

In some applications, we may not be interested in a multiplicity of DMT's, thus the diversity across different packets $b$ is dominated by the smallest diversity gain, i.e.,

$$
d_{S C}(r)=\min _{b} d(r, b)=(M-B+1)^{+}\left(1-\frac{B+1}{B} r\right)^{+}
$$

Note that in this expression, $B+1$ is a refresh cycle of the system, i.e., the period after which the source will transmit alone and will reset all the interferences at the relays. For an overall DMT above, since the two terms $M-B+1$ and $(B+1) / B$ move in opposite directions, one may optimize $B$ for each multiplexing gain $r$ so that the best diversity is obtained. This will lead to an overall DMT curve as shown in Figure 7.

\section{MHRS Protocol with Hybrid Joint Decoding}

In the previous section, we observed that successive cancellation in the MHRS protocol leads to error propagation and a gradual loss of diversity with increasing packet index. This loss arises from the reduced ability of the relays, after their own transmission, to correctly estimate and subtract the interference caused by other relays.

For better performance, we can employ a more powerful decoding technique at the relay. Whenever possible, the relays will decode by successive cancellation, but whenever that is not possible, the relays attempt an optimal joint decoding of the two arriving signals. Compare this with the method of Section IV-B, where the unavailable interfering signals were treated as noise. The more powerful method, denoted MHRS with hybrid joint decoding, improves the DMT of the MHRS protocol, and in fact meets the DMT upper bound up to a certain multiplexing gain, as we shall see in the sequel.

To calculate the DMT of MHRS with hybrid joint decoding, we use certain recent results on the so-called Z-channel. It is not difficult to see that our system model is a special case of the Z-channel, since the source is heard only by the relays, while the best relay in each interval is heard by both the destination and relays. (See Figure 8).

Recently, the DMT of the Z channel under general decoding was reported in [25]. Specializing the result of [25] to our channel model gives in the following relay outage diversity for a single-block decoding:

$$
d_{Z}(r)=(\min \{(1-r), 2(1-2 r)\})^{+}
$$

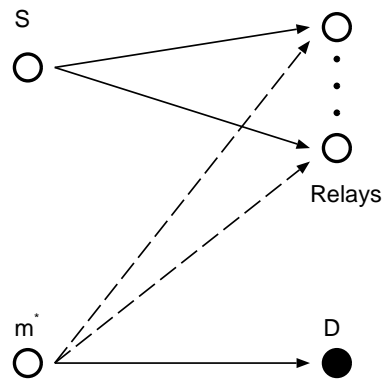

Fig. 8. The multiple $\mathrm{Z}$ channels arising from the MHRS channel model.

Since hybrid MHRS, for each packet detection, chooses the better of successive cancellation or joint decoding, the hybrid method must perform strictly better than either of its components. We will use this fact to find a lower bound to the DMT of the hybrid method.

The DMT of the non-hybrid method that always attempts successive cancellation was calculated in Section IV-B. In the following Lemma, we calculate the DMT of the non-adaptive method that always uses joint decoding.

Lemma 1: The DMT of MHRS protocol under joint decoding is lower bounded by

$$
\begin{aligned}
& d_{J D}(r)=\left(\operatorname { m i n } \left\{(M-1)\left(1-\frac{B+1}{B} r\right),\right.\right. \\
&\left.\left.\min _{t=0, \ldots, M-1}(2 M-2-t)-\frac{B+1}{B} r(4 M-4-3 t)\right\}\right)^{+}
\end{aligned}
$$

Proof: We first consider an individual frame. When all relays (excluding the selected one) use joint decoding for detecting the message of the source and using the DMT of the Z-channel given in (28), we can write

$$
\begin{aligned}
\operatorname{Pr}\{|D(s)|=t\} & \doteq\left(1-\rho^{-(\min \{(1-r), 2(1-2 r)\})^{+}}\right) \\
& \times \rho^{-(M-1-t)(\min \{(1-r), 2(1-2 r)\})^{+}} \\
& \doteq \rho^{-(M-1-t)(\min \{(1-r), 2(1-2 r)\})^{+}}
\end{aligned}
$$

Now, from (25), we have

$$
\operatorname{Pr}\left\{I_{m h r s}^{*}<R|| D(s) \mid\right\} \doteq \rho^{-t(1-r)^{+}}
$$

Therefore, the asymptotic outage probability is expressed as

$$
P_{\text {out }} \doteq \sum_{t} \rho^{-(t(1-r)+(M-1-t)(\min \{(1-r), 2(1-2 r)\}))^{+}}
$$

Hence, the diversity order is given by

$$
\begin{aligned}
d_{J D}(r)= & \left(\min _{t}\{\min [(M-1)(1-r), t(1-r)\right. \\
& +2(M-1-t)(1-2 r)]\})^{+} \\
= & (\min \{(M-1)(r-1), \\
& \left.\left.\min _{t=0, \ldots, M-1}(2 M-2-t)-r(4 M-4-3 t)\right\}\right)^{+}
\end{aligned}
$$




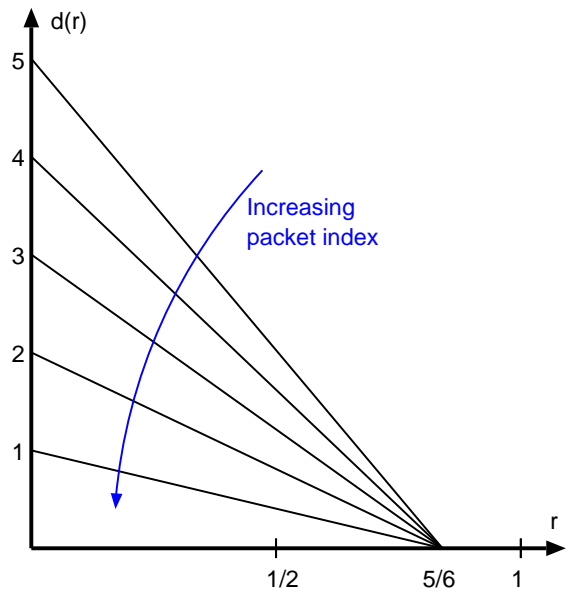

Fig. 6. Family of DMT's for various data positions in the transmission cycle of a 5-relay MHRS network, resulting in a variable error protection system.

We now consider the overall rate loss due to sending $B$ frames in $B+1$ time intervals, therefore we must make the substitution $r \rightarrow \frac{B+1}{B} r$. This completes the proof of the Lemma.

At low spectral efficiencies, the above expression shows a distinct improvement over successive cancellation.

The overall DMT of the hybrid joint decoding method is bounded by the following.

Theorem 4: The DMT of hybrid joint decoding is bounded below by:

$$
\begin{aligned}
& \max \left\{(M-B+1)^{+}\left(1-\frac{B+1}{B} r\right)^{+},\right. \\
& \min \left\{(M-1)\left(1-\frac{B+1}{B} r\right)^{+},\right. \\
& \left.\left.\min _{t=0, \ldots, M-1}\left[(2 M-2-t)-\frac{B+1}{B} r(4 M-4-3 t)\right]^{+}\right\}\right\}
\end{aligned}
$$

Proof: The DMT of the adaptive (hybrid) protocol $d(r)$ is bounded below by the two DMT's belonging to the pure successive cancellation and joint decoding methods $d_{S C}(r), d_{J D}(r)$. It immediately follows that $d(r) \geq$ $\max \left\{d_{S C}(r), d_{J D}(r)\right\}$.

Figure 9 compares the diversity-multiplexing tradeoff of several DF-based protocols in a two-hop relay network with $M=10$ and $B=6$. Also, shown the MHRS protocol upper bound derived in Section IV-A. The hybrid MHRS protocol attains better DMT performance, across a large range of spectral efficiencies, compared with distributed space-time codes and opportunistic relaying ${ }^{2}$.

Remark 5: The hybrid MHRS protocol, as analyzed above, used a successive cancellation component whose minimal DMT was included in the analysis. The reader may recall, however, that the successive cancellation MHRS produces a family of unequal error protection DMT's. Thus, the hybrid strategy can also inherit the embedded DMT property of the successive cancellation. Since the hybrid DMT is influenced

\footnotetext{
${ }^{2}$ Distributed space-time codes and opportunistic relaying have the same DMT, for compactness, only one of them is marked in Figure 9.
}

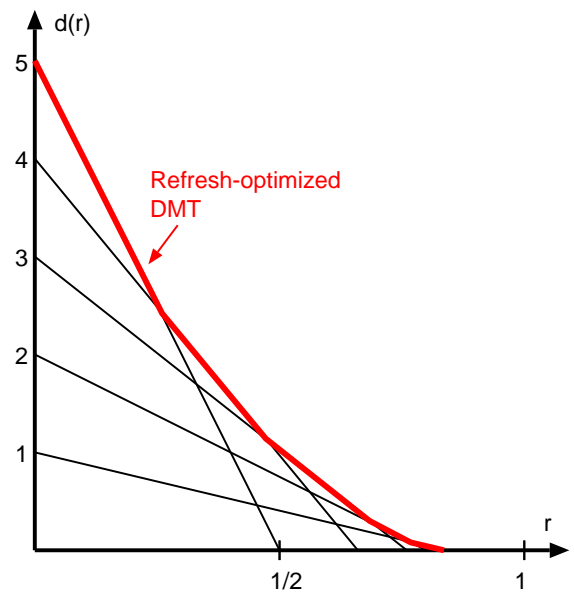

Fig. 7. The DMT of the overall MHRS with five relays, where the refresh cycle is optimized separately for each $r$.

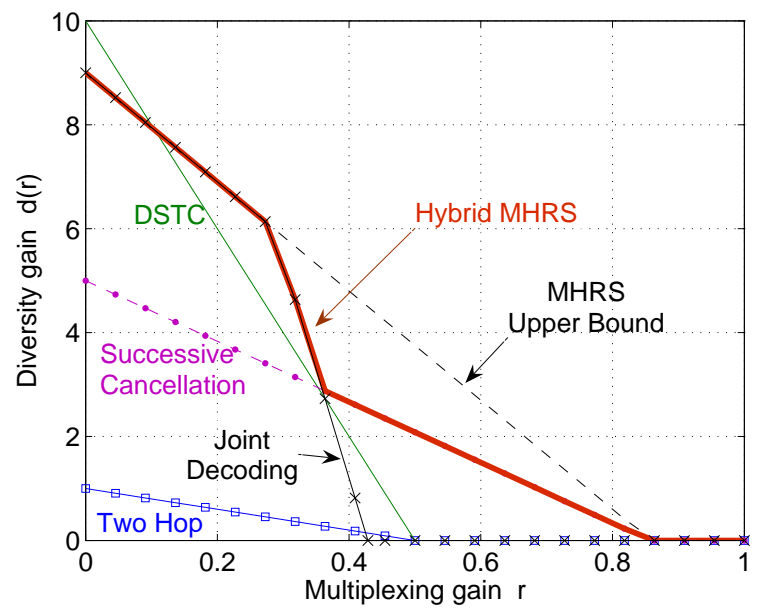

Fig. 9. Diversity-multiplexing tradeoff of different protocols in a two-hop network with ten relays.

by the successive cancellation DMT in the high-rate regime, it follows that the embeddedness of the hybrid DMT is observable at high multiplexing rates. In the low multiplexing rates, all frames will experience the same diversity. To summarize, the embedded hybrid MHRS has the following achievable DMT, where $b$ is the index of the packet.

$$
\begin{aligned}
& \max \left\{(M-b+1)^{+}\left(1-\frac{B+1}{B} r\right)^{+},\right. \\
& \min \left\{(M-1)\left(1-\frac{B+1}{B} r\right)^{+},\right. \\
&\left.\left.\min _{t=0, \ldots, M-1}\left[(2 M-2-t)-\frac{B+1}{B} r(4 M-4-3 t)\right]^{+}\right\}\right\}
\end{aligned}
$$

\section{CONClusion}

This paper proposes spectrally-efficient relay selection techniques with limited feedback. In a multi-relay scenario, when a direct link exists between the source and destination, we propose an Incremental Transmission Relay Selection (ITRS) 
protocol. In the absence of a direct link, we propose a MultiHop Relay Selection (MHRS) protocol. The ITRS achieves the MISO DMT bound, and a bound is developed for MHRS which is tight over a portion of the multiplexing gains. Both improve over existing methods for half-duplex DF relay systems.

We can foresee several avenues for future research. One of them, which has a strong practical impact, is the design of channel codes for the above-mentioned protocols. Another point of interest is power/rate control strategies across the nodes while maintaining limited feedback. A code-combining type-II H-ARQ for the ITRS protocol may also provide improved performance. Finally, design issues related to higher layers can be explored, including selection of the number of transmission blocks for MHRS and designing signaling protocols for exchanging the feedback information over a control channel.

\section{APPENDIX I}

\section{ITRS OUTAGE ANALYSIS}

Computing the outage probability of ITRS hinges upon a closed-form expression for

$$
\operatorname{Pr}\left\{\frac{1}{2} \log \left[1+\rho\left(g_{s, d}+g_{m^{*}, d}\right)\right] \leq \frac{R}{2} \mid D(s)\right\}
$$

whose calculation is the main goal of this appendix.

Lemma 2: Consider an exponential random variable $U$ with mean $\lambda_{u}$, and a random variable $V$ which is maximum of a group of $t$ i.i.d. exponential random variables, each with mean $\lambda_{g}$. The PDF of $W=U+V$ is given by

$$
\begin{aligned}
f_{W}(w)=t & \exp \left(-\frac{w}{\lambda_{u}}\right) \sum_{k=0}^{t-1}\left(\begin{array}{c}
t-1 \\
k
\end{array}\right) \frac{(-1)^{k}}{(k+1) \lambda_{u}-\lambda_{g}} \\
& \left(1-\exp \left[-\left(\frac{k+1}{\lambda_{g}}-\frac{1}{\lambda_{u}}\right) w\right]\right)
\end{aligned}
$$

Proof:

$$
f_{U}(u)=\frac{1}{\lambda_{u}} \exp \left(-\frac{u}{\lambda_{u}}\right)
$$

The CDF of a maximum of i.i.d. exponential random variables is given by:

$$
F_{V}(v)=\left(1-\exp \left(-\frac{v}{\lambda_{g}}\right)\right)^{t}
$$

Differentiating with respect to $v$, we get

$$
f_{V}(v)=\frac{t}{\lambda_{g}} \exp \left(-\frac{v}{\lambda_{g}}\right)\left(1-\exp \left(-\frac{v}{\lambda_{g}}\right)\right)^{t-1}
$$

Using the convolution integral, the PDF of $W$ is given by:

$$
\begin{gathered}
f_{W}(w)=\frac{t}{\lambda_{g} \lambda_{u}} \exp \left(-\frac{w}{\lambda_{u}}\right) \int_{0}^{w} \exp \left(-\frac{v}{\lambda_{e q}}\right) \\
\left(1-\exp \left(-\frac{v}{\lambda_{g}}\right)\right)^{t-1} d v
\end{gathered}
$$

where we have defined:

$$
\frac{1}{\lambda_{e q}} \triangleq \frac{1}{\lambda_{g}}-\frac{1}{\lambda_{u}}
$$

Using the binomial expansion, performing the integration and simplifying, one obtains,

$$
\begin{aligned}
f_{W}(w)=t & \exp \left(-\frac{w}{\lambda_{u}}\right) \sum_{k=0}^{t-1}\left(\begin{array}{c}
t-1 \\
k
\end{array}\right) \frac{(-1)^{k}}{(k+1) \lambda_{u}-\lambda_{g}} \\
& \left(1-\exp \left[-\left(\frac{k+1}{\lambda_{g}}-\frac{1}{\lambda_{u}}\right) w\right]\right)
\end{aligned}
$$

Now, let $U=g_{s, d}, V=g_{m^{*}, d}$ and $W=U+V$. Then, the PDF of $W$ is given by:

$$
\begin{aligned}
f_{W}(w)=t & \exp \left(-\frac{w}{\lambda_{s, d}}\right) \sum_{k=0}^{t-1}\left(\begin{array}{c}
t-1 \\
k
\end{array}\right) \frac{(-1)^{k}}{(k+1) \lambda_{s, d}-\lambda_{m, d}} \\
& \left(1-\exp \left[-\left(\frac{k+1}{\lambda_{m, d}}-\frac{1}{\lambda_{s, d}}\right) w\right]\right)
\end{aligned}
$$

The CDF of this expression can be obtained via integration;

$$
\begin{aligned}
F_{W}(\gamma)=t & \sum_{k=0}^{t-1}\left(\begin{array}{c}
t-1 \\
k
\end{array}\right) \frac{(-1)^{k}}{(k+1) \lambda_{s, d}-\lambda_{m, d}} \\
& \times \int_{0}^{\gamma} \exp \left(-\frac{w}{\lambda_{s, d}}\right) \\
& \left(1-\exp \left[-\left(\frac{k+1}{\lambda_{m, d}}-\frac{1}{\lambda_{s, d}}\right) w\right]\right) d w
\end{aligned}
$$

where as previously defined, $\gamma=\frac{2^{R}-1}{\rho}$. Also, to simplify the calculations, we let $\lambda_{s, d}=\lambda_{m^{*}, d}=\frac{1}{\mu}$. After integration by parts and collecting terms, whose details are omitted for brevity, we obtain:

$$
\begin{aligned}
F_{W}(\gamma)= & {\left[t \sum _ { k = 1 } ^ { t - 1 } ( \begin{array} { c } 
{ t - 1 } \\
{ k }
\end{array} ) \frac { ( - 1 ) ^ { k } } { k } \left(1+\frac{\exp (-\mu(k+1) \gamma)-1}{(k+1)}\right.\right.} \\
& -\exp (-\mu \gamma))]+t(1-(\mu \gamma+1) \exp (-\mu \gamma))
\end{aligned}
$$

Finally, the overall outage probability for ITRS protocol is calculated by

$$
\begin{aligned}
P_{\text {out }, \text { ITRS }}= & \sum_{D(s)} F_{W}(\gamma) \times\left(\begin{array}{c}
M \\
t-1
\end{array}\right) \exp \left(-\frac{\gamma}{\lambda_{s, m}}\right)^{t-1} \\
& \left(1-\exp \left(-\frac{\gamma}{\lambda_{s, m}}\right)\right)^{M-t+1}
\end{aligned}
$$

\section{APPENDIX II}

\section{ITRS DMT ANALYSIS}

The effective rate of the ITRS protocol is $\eta$, as defined in (12), therefore the multiplexing gain must be defined with respect to $\eta$. However, one can equivalently use the nominal per-packet transmission rate $R$ for the DMT calculations, since an inspection of (12) shows they are asymptotically equivalent:

$$
\lim _{\rho \rightarrow \infty} \eta=R
$$

Hence we can proceed and calculate the multiplexing gain based on $R$ as defined in (1).

There are two ways to obtain the diversity order in terms of the multiplexing gain. One can either find an upper bound 
on the outage expression in (8) in a manner similar to [1, Theorem 3], or use the closed-form outage expression that is developed in this paper, in asymptotic SNR form. The former approach is easier and we briefly mention the required steps.

First, from (9), at high SNR

$$
\operatorname{Pr}\{|D(s)|=t\} \doteq \rho^{(r-1)(M-t+1)}\left(\frac{1}{\lambda_{s, m}}\right)^{M-t+1}
$$

Now, from (7), at high SNR

$$
\begin{aligned}
& \operatorname{Pr}\left\{I_{i t r s}^{*}<\frac{r \log \rho}{2} \mid D(s)\right\}= \operatorname{Pr}\left\{\log \left(1+\rho\left(g_{s, d}+g_{m^{*}, d}\right)\right)\right. \\
&\leq r \log \rho \mid D(s)\} \\
& \leq \operatorname{Pr}\left\{g_{s, d} \leq \rho^{r-1} \mid D(s)\right\} \\
& \times \operatorname{Pr}\left\{g_{m^{*}, d} \leq \rho^{r-1} \mid D(s)\right\} \\
& \doteq \rho^{r-1} \rho^{t(r-1)} \\
&= \rho^{(t+1)(r-1)}
\end{aligned}
$$

where we have used the results of Lemmas 2 and 3 of [1]. Combining (49) and (50), the diversity order of the ITRS protocol is given by

$$
d_{I T R S}(r)=(M+2)(1-r)^{+}
$$

\section{REFERENCES}

[1] A. Bletsas, A. Khisti, D. P. Reed, and A. Lippman, "A simple cooperative diversity method based on network path selection," IEEE J. Select. Areas Commun., vol. 24, no. 3, pp. 659-672, Mar. 2006.

[2] D. J. Love, R. W. Heath, W. Santipach, and M. L. Honig, "What is the value of limited feedback for MIMO channels?" IEEE Commun. Mag., vol. 42, no. 10, pp. 54-59, Oct. 2004.

[3] B. Zhao and M. C. Valenti, "Practical relay networks: a generalization of hybrid-ARQ," IEEE J. Select. Areas Commun., vol. 23, no. 1, pp. 7-18, Jan. 2005.

[4] J. N. Laneman and G. W. Wornell, "Distributed space-time-coded protocols for exploiting cooperative diversity in wireless networks," IEEE Trans. Inform. Theory, vol. 49, no. 10, pp. 2415-2425, Oct. 2003.

[5] K. Azarian, H. El Gamal, and P. Schniter, "On the achievable diversitymultiplexing tradeoff in half-duplex cooperative channels," IEEE Trans. Inform. Theory, vol. 51, no. 12, pp. 4152-4172, Dec 2005.

[6] A. Tajer and A. Nosratinia, "Opportunistic cooperation via relay selection with minimal information exchange," in Proc. IEEE International Symposium on Information Theory (ISIT), Nice, France, June 2007.

[7] A. Bletsas, H. Shin, and M. Z. Win, "Cooperative communications with outage-optimal opportunistic relaying," IEEE Transactions on Wireless Communications, vol. 6, no. 9, pp. 3450-3460, Sep. 2007.

[8] A. Nosratinia and T. E. Hunter, "Grouping and partner selection in cooperative wireless networks," IEEE J. Select. Areas Commun., vol. 54, no. 4, pp. 760-378, Feb. 2006.

[9] Z. Lin, E. Erkip, and A. Stefanov, "Cooperative regions and partner choice in coded cooperative systems," IEEE Trans. Commun., vol. 54, no. 7, pp. 1323-1334, July 2006.

[10] E. Beres and R. Adve, "Selection cooperation in multi-source cooperative networks," IEEE Transactions on Wireless Communications, vol. 7, no. 1, pp. 118-127, Jan 2008.

[11] A. Bletsas, H. Shin, and M. Z. Win, "Outage optimality of opportunistic amplify-and-forward relaying," IEEE Communication Letters, vol. 11, no. 3, pp. 261-263, March 2007.

[12] Y. Zhao, R. Adve, and L. T. J, "Improving amplify-and-forward relay netwroks: Optimal power allocation versus selection," IEEE Transactions on Wireless Communications, vol. 6, no. 8, pp. 3114-3123, Aug. 2007.

[13] — "Symbol error rate of selection amplify-and-forward relay systems," IEEE Communication Letters, vol. 10, no. 11, pp. 757-759, Nov. 2006.
[14] I. Krikidis, J. Thompson, S. McLaughlin, and N. Goertz, "Amplifyand-forward with partial relay selection," IEEE Communication Letters, vol. 12, no. 4, pp. 235-237, April 2008.

[15] J. Cai, X. Shen, J. W. Mark, and A. S. Alfa, "Semi-distributed user relaying algorithm for amplify-and-forward wireless relay netwroks," IEEE Transactions on Wireless Communications, vol. 7, no. 4, pp. 13481357, April 2008.

[16] D. S. Michalopoulos, G. K. Karagiannidis, T. A. Tsiftsis, and R. K. Mallik, "Distributed transmit antenna selection (DTAS) under performance or energy consumption constraints," IEEE Transactions on Wireless Communications, vol. 7, no. 4, pp. 1168-1173, April 2008.

[17] C. K. Lo, R. W. Heath, and S. Vishwanath, "Opportunistic relay selection with limited feedback," in Proc. IEEE Vehicular Technology Conference (VTC), April 2007, pp. 135 - 139.

[18] S. Yang and J.-C. Belfiore, "Towards the optimal amplify-and-forward cooperative diversity scheme," IEEE Trans. Inform. Theory, vol. 53, no. 9, pp. 3114-3126, Sep. 2007.

[19] L. H. Ozarow, S. Shamai, and A. D. Wyner, "Information theoretic considerations for cellular mobile radio," IEEE Trans. Vehicular Techonolgy, vol. 43, no. 2, pp. 359-378, May 1994.

[20] L. Zheng and D. N. C. Tse, "Diversity and multiplexing: a fundamental tradeoff in multiple-antenna channels," IEEE Trans. Inform. Theory, vol. 49, no. 5, pp. 1073-1096, May 2003.

[21] J. N. Laneman, D. N. C. Tse, and G. W. Wornell, "Cooperative diversity in wireless networks: Efficient protocols and outage behavior," IEEE Trans. Inform. Theory, vol. 50, no. 12, pp. 3062-3080, Dec. 2004.

[22] H. El Gamal, G. Caire, and M. O. Damen, "The MIMO ARQ channel: Diversity-multiplexing-delay tradeoff," IEEE Trans. Inform. Theory, vol. 52, no. 8, pp. 3601-3621, Aug. 2006.

[23] M. Yuksel and E. Erkip, "Cooperative wireless systems: A diversity multiplexing tradeoff perspective," IEEE Trans. Inform. Theory, vol. 53, no. 10, pp. 3371 - 3393, Oct 2007.

[24] Y. Jiang and M. K. Varanasi, "Diversity-multiplexing gain tradeoff of MIMO systems with antenna selection," in Proc. IEEE International Symposium on Information Theory (ISIT), Nice, France, June 2007.

[25] M. Ansari, A. Bayesteh, and A. K. Khandani, "Diversity-multiplexing tradeoff in Z-channel," in Proc. 10th Canadian Workshop on Information Theory (CWIT), June 2007, pp. 21-24.

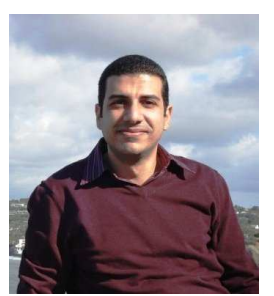

Ramy Tannious [S'04] received the B.Sc. (with honors) and M.Sc. degrees in electrical engineering from Alexandria University, Egypt, in 1999 and 2003, respectively. He is currently working toward the $\mathrm{Ph} . \mathrm{D}$. degree in electrical engineering at the University of Texas at Dallas. His research interests include network information theory, wireless relay networks, MIMO communications, and multi-user MIMO systems.

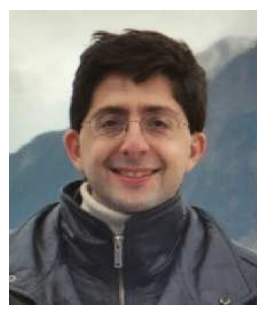

Aria Nosratinia [M'97, SM'04] is professor of Electrical Engineering at the University of Texas at Dallas. He received his $\mathrm{PhD}$ in Electrical and Computer Engineering from the University of Illinois at Urbana-Champaign in 1996. He has held visiting positions at Princeton University, Rice University, and UCLA. His research interests are in the general area of signal processing, coding and information theory, with applications to wireless communications. He currently serves as editor for the IEEE Transactions on Information Theory, IEEE Transactions on Wireless Communications, IEEE Signal Processing Letters, and IEEE Wireless Communications, and in 2002-2008 has served as editor for the IEEE Transactions on Image Processing. He is a recipient of the National Science Foundation career award, as well as two chapter awards for service to the IEEE Signal Processing Society. 